\title{
Correction of the deviated tip and columella in crooked nose
}

\author{
Man-Koon Suh \\ JW Plastic Surgery Center, Seoul, Korea
}

The primary procedural components of deviated nose correction are as follows: osteotomy to correct bony deviation, septal deviation correction, manipulation of the dorsal septum to correct upper lateral cartilage deviation, and correction of functional problems (manipulation for correction of internal valve collapse and hypertrophy of the inferior turbinate). The correction of tip and nostril asymmetry cannot be overemphasized, because if tip and nostril asymmetry is not corrected, patients are unlikely to provide favorable evaluations from an aesthetic standpoint. Tip asymmetry, deviated columella, and resulting nostril asymmetry are primarily caused by lower lateral cartilage problems, which include deviation of the medial crura, discrepancy in the height of the medial crura, and asymmetry or deformity of the lateral crura. However, caudal and dorsal septal deviation, which is a more important etiology, should also be corrected. A columellar strut graft, correction of any discrepancy in the height of the medial crura, or lateral crural correction is needed to correct lower lateral cartilage deformation depending on the type. In order to correct caudal septal deviation, caudal septal shortening, repositioning, or the cut-and-suture technique are used. Surgery to correct dorsal septal deviation is performed by combining a scoring and splinting graft, a spreader graft, and/or the clocking suture technique. Moreover, when correcting a deviated nose, correction of asymmetry of the alar rim and alar base should not be overlooked to achieve tip and nostril symmetry.

Keywords Acquired nose deformity / Nasal septum / Rhinoplasty

\author{
Correspondence: Man-Koon Suh \\ JW Plastic Surgery Center, 553 \\ Samseong-ro, Gangnam-gu, Seoul \\ 06155, Korea \\ Tel: $+82-541-5114$ \\ Fax: +82-541-5112 \\ E-mail:smankoon@hanmail.net
}

This review article was prepared by the Korean Academic Association of Rhinoplasty Surgeons (KAARS).

Received: September 5, $2020 \bullet$ Revised: October 4, $2020 \bullet$ Accepted: October 4, 2020

pISSN: 2234-6163 • elSSN: 2234-6171 • https://doi.org/10.5999/aps.2020.01774• Arch Plast Surg 2020;47:495-504

\section{INTRODUCTION}

\begin{abstract}
A deviated nose, also known as a crooked nose, is defined as a condition in which the nose does not follow the mid-vertical line of the face. A deviated nose poses both functional and aesthetic problems, the former of which is exemplified by nasal obstruction [1]. Correction of nasal deviation is among the most challenging aspects of rhinoplasty and surgeons should explore functional and aesthetic solutions.

A deviated nose involves deviation in the nasal bone, the septum, and the upper and lower lateral cartilage (Fig. 1) [2].
\end{abstract}

Therefore, the primary procedural components of deviated nose correction are as follows: osteotomy to correct bony deviation, septal deviation correction, manipulation of the dorsal septum to correct upper lateral cartilage deviation, and correction of functional problems (manipulation for correction of internal valve collapse and hypertrophy of the inferior turbinate) [3].

Many studies on deviated nose correction have covered operative techniques involving the aforementioned procedures. Simultaneously, because almost all deviated noses involve a deviated tip, oblique columella, or asymmetry of the nostrils (Fig. 2), it is difficult to achieve an optimal result in deviated nose correc- 


\section{Fig. 1. Anatomy of deviated nose}

(A) Deviated nasal bone and upper lateral cartilage (ULC), and asymmetric lower lateral cartilage (LLC). (B) Deviated septum.
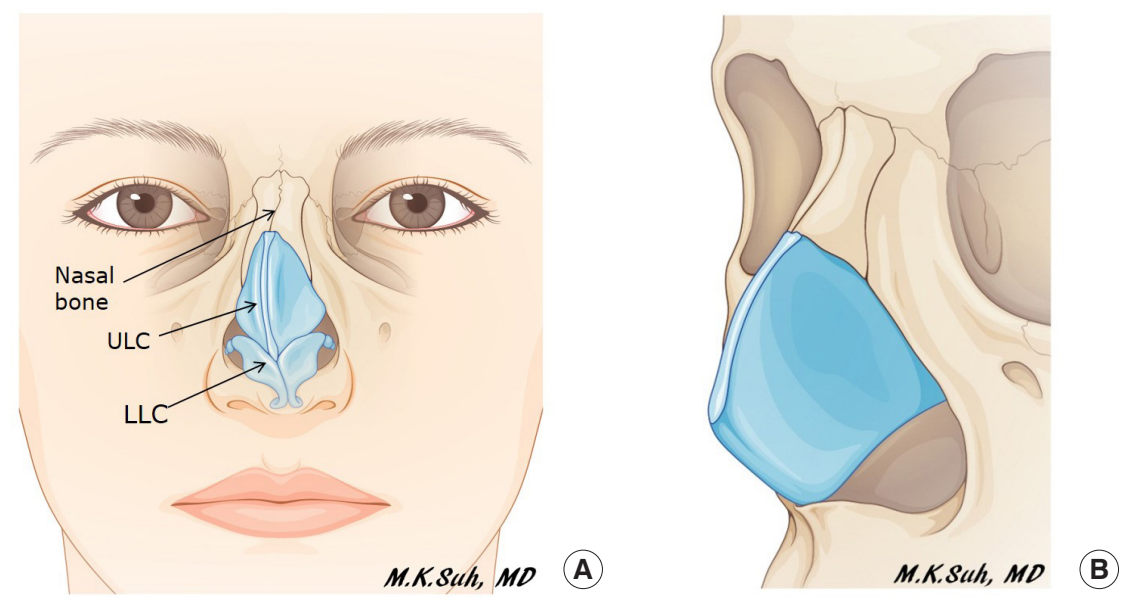

Fig. 2. Deviated tip/columella

A deviated nose is always accompanied by a deviated tip/columella (A) and nostril asymmetry (B).
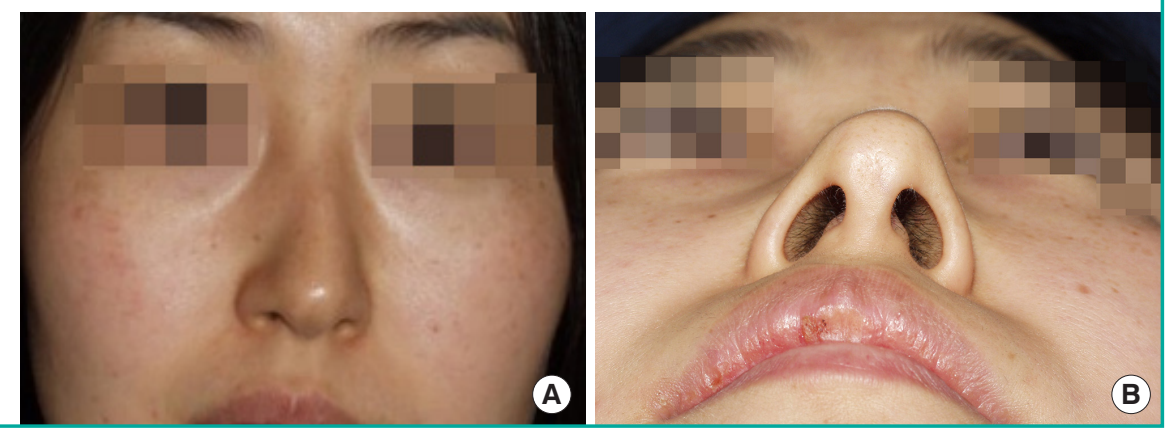

Fig. 3. Types of lower lateral cartilage deformities

(A) Twisted medial crura. (B) Height discrepancy of the medial crura. The right medial crus is longer than the left. (C) Deformed lateral crura.
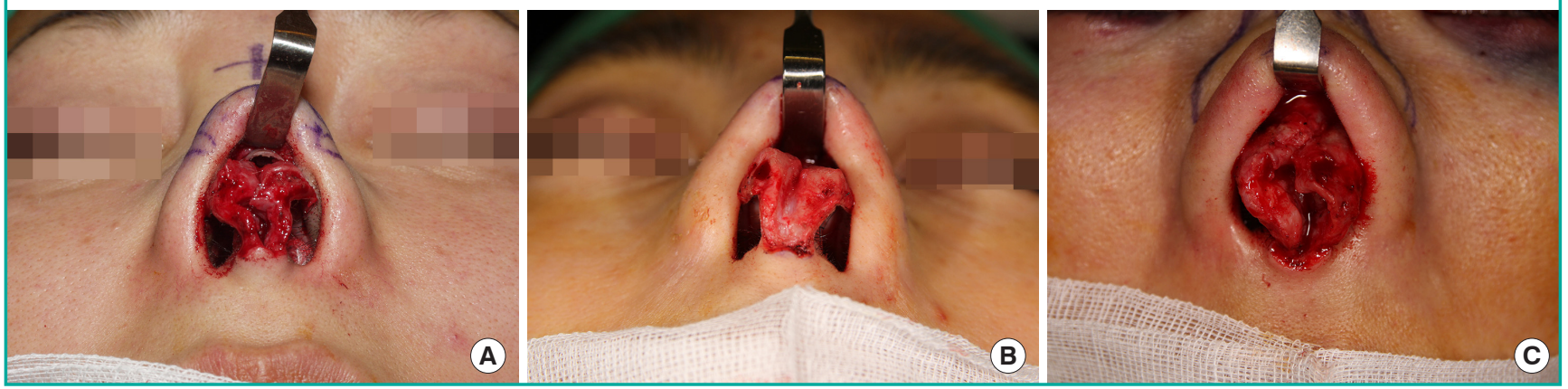

tion without correcting those problems [4].

A symmetric tip and nostril is a criterion for a beautiful nose. When there is tip, columella, or nostril asymmetry because these areas have been incompletely corrected, the patient is likely to be dissatisfied and the surgeon's reputation will suffer. Therefore, surgeons should focus on deviation of the tip and columella, as well as the correction of bony and mid-vault framework deviation.

\section{CAUSES OF DEVIATION OF THE TIP/COLUMELLA AND ASYMMETRY OF THE NOSTRILS}

Tip asymmetry, deviated columella, and the resultant nostril asymmetry are primarily caused by lower lateral cartilage problems. Examples of lower lateral cartilage problems include deviation of the medial crura, a discrepancy in the height of the medial crura, and asymmetry or deformity of the lateral crura (Fig. 3). 
However, asymmetry of the tip and nostril does not merely arise from asymmetric lower lateral cartilages. A deviated septum is a more common cause, and the majority of cases involving deviation of the tip/columella and asymmetry of the nostrils show a close relationship with deviation of the caudal and dorsal septum.

The mechanism through which caudal septal deviation causes columella deviation is that the deviated caudal septum moves the medial crura laterally (Fig. 4) [4]. A deviated dorsal septum contributes to tip and columella deviation by laterally moving the dome in the lower lateral cartilage and lateral crura (Fig. 4) [4].

Therefore, when performing correction of columella and nostril asymmetries, the surgeon should correct the caudal and dorsal septum, as well as the asymmetry of the lower lateral cartilage.

Finally, a unilateral retracted ala rim, an asymmetric alar rim, or asymmetry of the width of the alar base can cause nostril asymmetry (Fig. 5).

\section{Fig. 4. Asymmetric lower lateral cartilage in deviated nose}

The deviated upper lateral cartilage pushes the lateral crus laterally, while the deviated caudal septum pushes out the medial crus (white arrows).

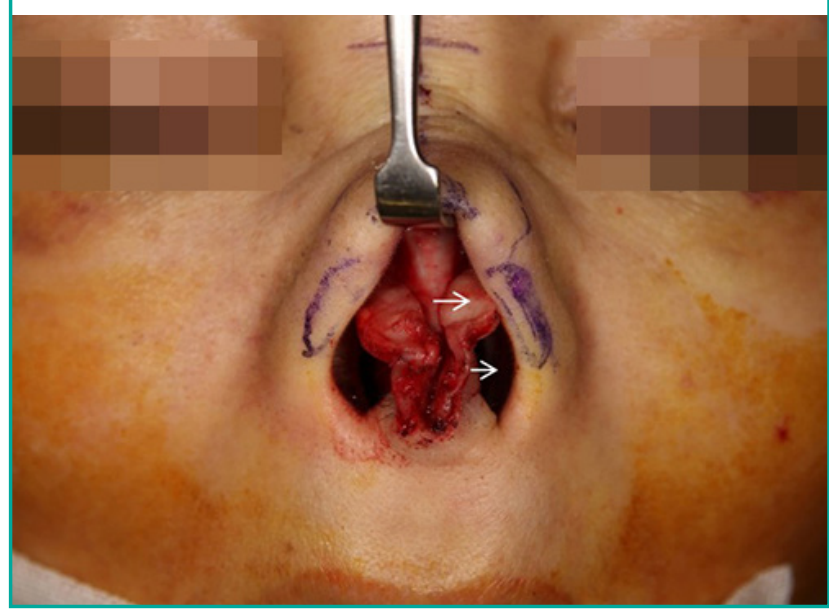

\section{OPERATIVE TECHNIQUES FOR DEVIATION OF THE TIP/ COLUMELLA AND ASYMMETRY OF THE NOSTRILS IN DEVIATED NOSE}

\section{Correction of lower lateral cartilage problems}

When deviation or twisting of the medial crura is the cause of a deviated columella or asymmetric tip, the definitive correction is to straighten the medial crura using a columellar strut graft (Fig. 6) [5].

The septum is preferred as the donor of the columellar strut graft because it is possible to obtain the donor cartilage when septal correction is performed as part of deviated nose correction. If a surgeon considers that it is inappropriate to use septal cartilage, conchal cartilage can be used. The cymba conchae is a more preferable donor site because it is longer than the cavum conchae.

When performing a columellar strut graft, the nostril apex is the most important reference point to make a symmetric nostril [4]. The surgeon should fix the medial crura to the columellar strut graft with the apex of the nostril located on both sides of a single horizontal line. In this way, the same nostril height of left and right can be obtained, and any lingering asymmetry of the alar dome or lateral crura can easily be corrected by suturing or segmental resection (Fig. 7). On the contrary, if the position of the dome, instead of the nostril height, is used as a reference point and is adjusted first, the remaining asymmetry of nostril height is not easily corrected.

Differences in the height of the medial crura on both sides cause nostril asymmetry because the heights of the nostril apex on the left and right become different. In order to correct this, shortening of the longer medial crus or elongation of the shorter medial crus needs to be performed.

\section{Shortening of the longer medial crus}

After cutting a columellar segment of the longer medial crus, an

Fig. 5. Nostril asymmetry

Nostril asymmetry resulting from retracted ala of the left side $(A)$ and asymmetry of alar base width (B).
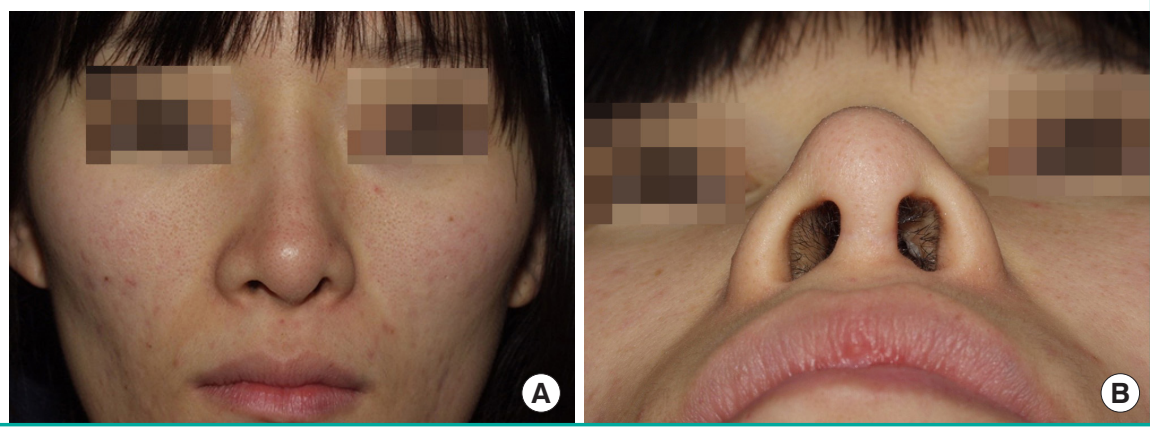
Fig. 6. Correction of twisted medial crura

(A) Nostril asymmetry and columella deviation. (B) The twisted medial crura is the main cause of nostril asymmetry and deviated columella. (C) Columellar strut graft using septal cartilage. (D) Symmetric nostrils and a straight columella were achieved.
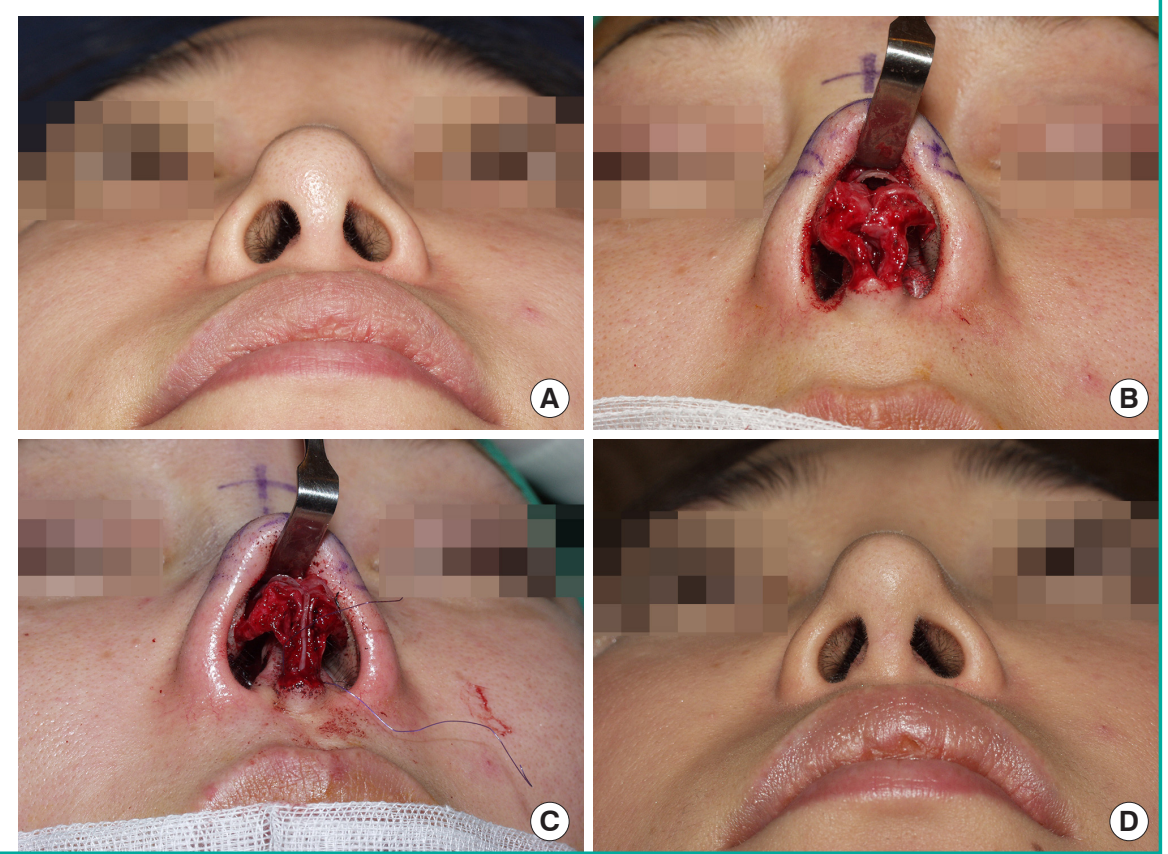

Fig. 7. Reference point for nostril symmetry

(A) Deviated columella and asymmetric nostrils. (B) A columellar strut graft being performed. The nostril apexes are at the same height. (C) The right lateral crus is shortened to make symmetric lateral crura. (D) Both nostril apexes and lateral crura achieved symmetry. (E) Postoperative results showing symmetric nostrils and a straight columella.
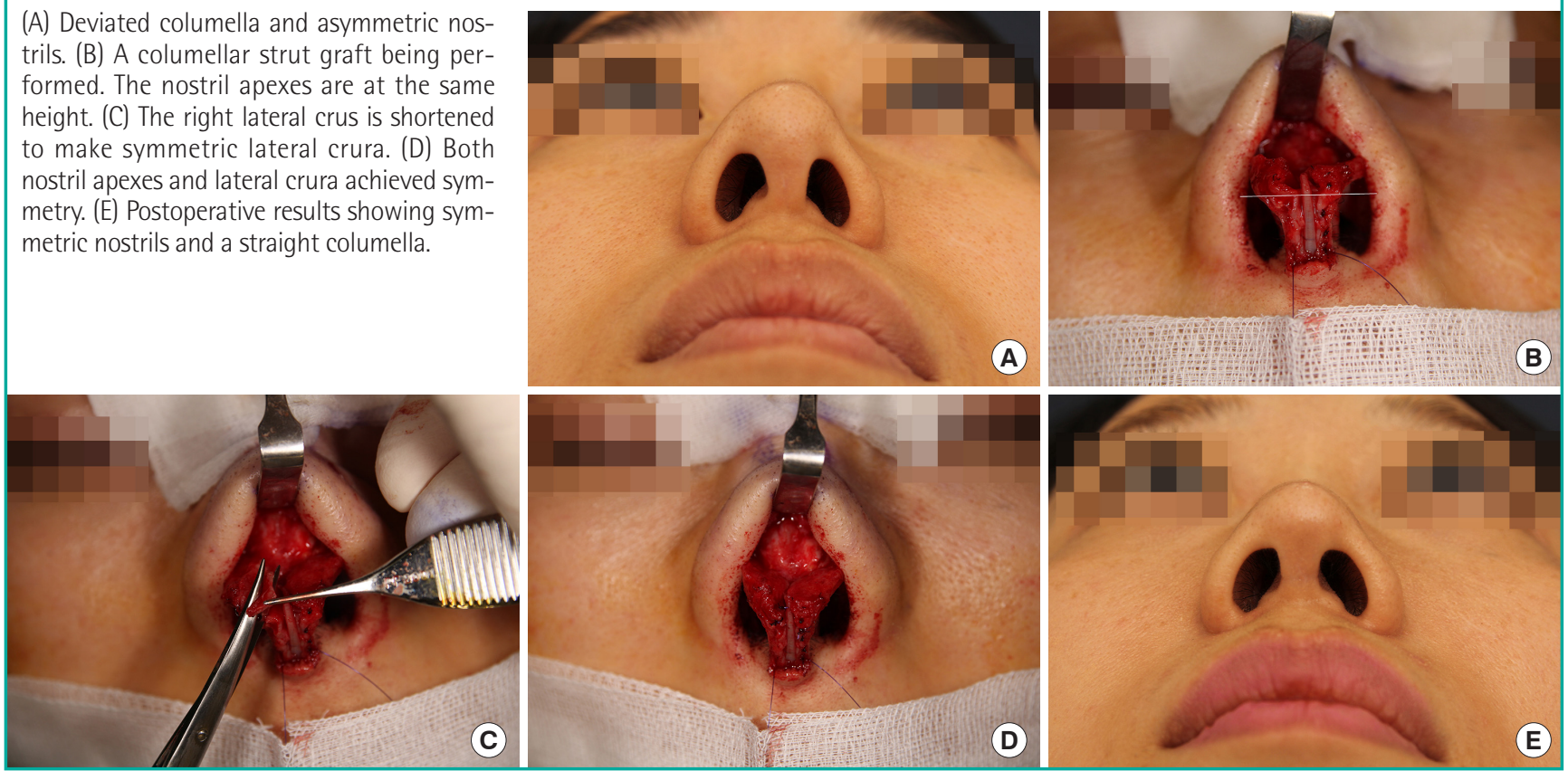

overlapping suture is performed (Fig. 8).

\section{Lengthening of shorter medial crus}

A gap is made by excising a columellar segment in the shorter medial crus, and a columella strut graft is performed (Fig. 9). Deformed lateral crura or asymmetric lateral crura also contribute to tip asymmetry and columella deviation.

\section{Buckling of the lateral crura}

A concavity control suture can be used to correct mild buckling of the lateral crura (Fig. 10) [6]. Severe cases may necessitate a lateral crural onlay graft.

\section{Asymmetry in the length of the lateral crura}

Caudal displacement of the lateral crus, which detaches from the hinge complex on the shorter side, or shortening of the lat- 


\section{Fig. 8. Shortening of the longer medial crus}

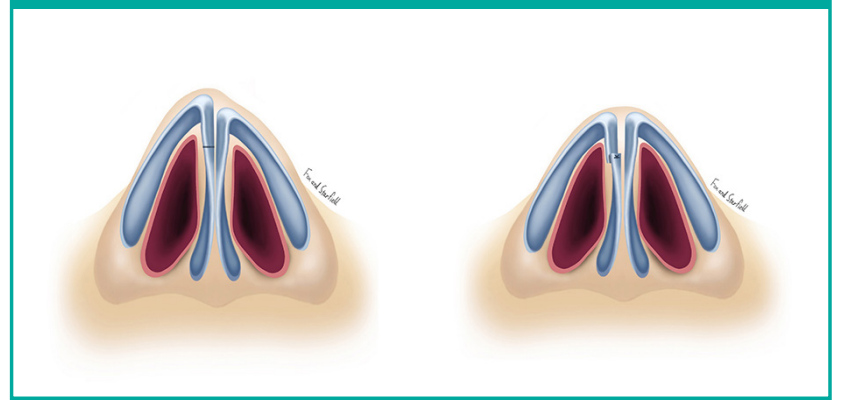

Fig. 9. Lengthening of the shorter medial crus

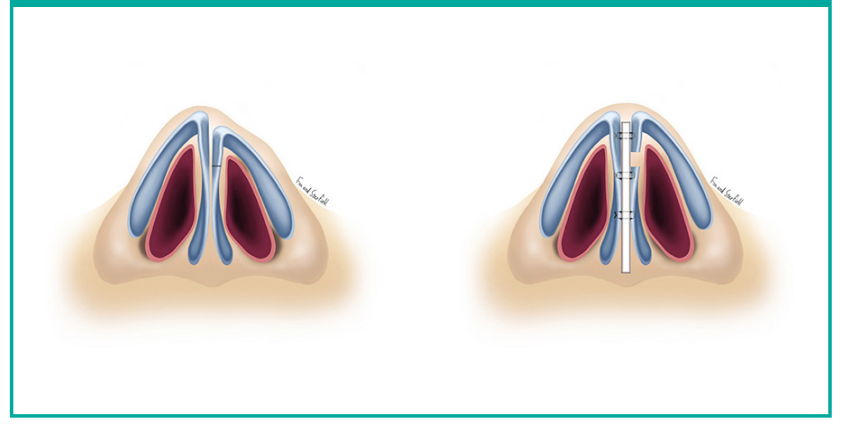

Fig. 10. Concavity control suture
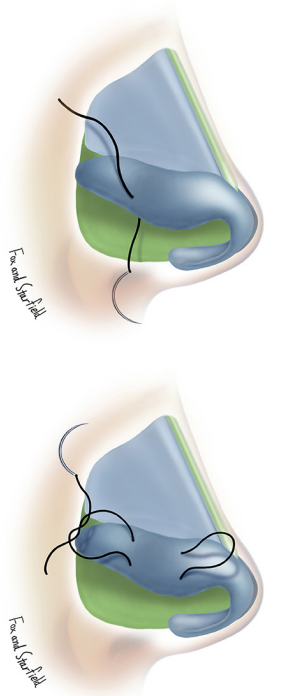

eral crus on the longer side is performed. Shortening of the lateral crus is carried out at the lateral-most part of the lateral crus using the cut-and-overlapping suture technique (Fig. 11).

\section{Correction of deviation of the caudal and dorsal septum}

Correcting deviation of the caudal and dorsal septum is a vitally important part of surgery to correct a deviated tip and columella.

\section{Fig. 11. Shortening of the longer lateral crus}
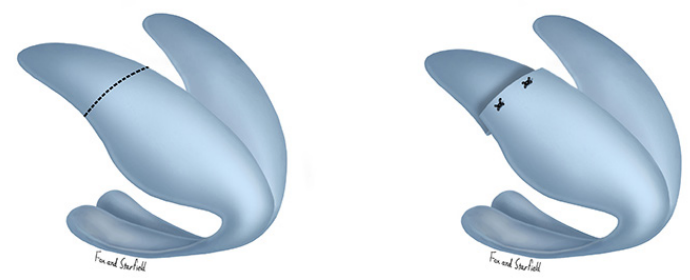

Fig. 12. Deviated caudal septum from excess length

Caudal septal deviation in which the caudal septum is on the anterior nasal spine, while the caudal septal cartilage has excess length.
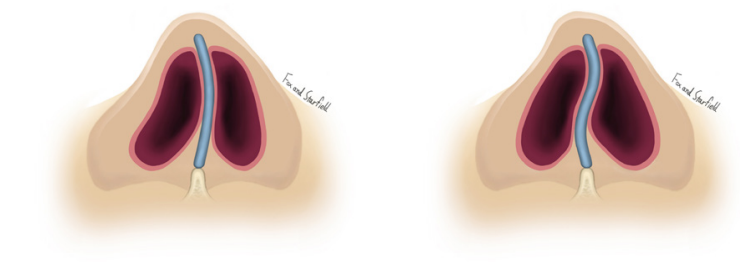

\section{Correction of deviation of the caudal septum}

Anteroposterior deviation of the caudal septum is corrected as follows.

In one type, the anterior nasal spine (ANS) is placed on the midline and the caudal septum is located on the ANS, and the caudal septal cartilage is deviated in a $\mathrm{C}$ or $\mathrm{S}$ pattern because of its excess length (Fig. 12).

Correction of this type of deviation requires caudal septal shortening, and can be performed through segmental resection of the caudal septum [7] or the cut-and-suture technique [8].

Segmental resection of caudal septal cartilage

After dislocating the posterior end of the caudal L-strut from the maxillary crest and the ANS, segmental resection of the excess length is performed [7]. A shortened caudal L-strut is then fixed to the periosteum of the ANS (Figs. 13, 14). A figure-ofeight suture is used to perform fixation of the caudal end of the caudal septal cartilage with the soft tissue of the ANS. Therefore, enough soft tissue should be left on the ANS when performing dissection. If the concave curvature in the cauda septum does not disappear spontaneously even after segmental resection, scoring should be done on the concave side and a splinting batten graft should be applied.

Cut-and-suture technique

A deviated caudal septal strut is cut at the most convex part, after which the cut ends are overlapped and sutured together (Fig. 15). 

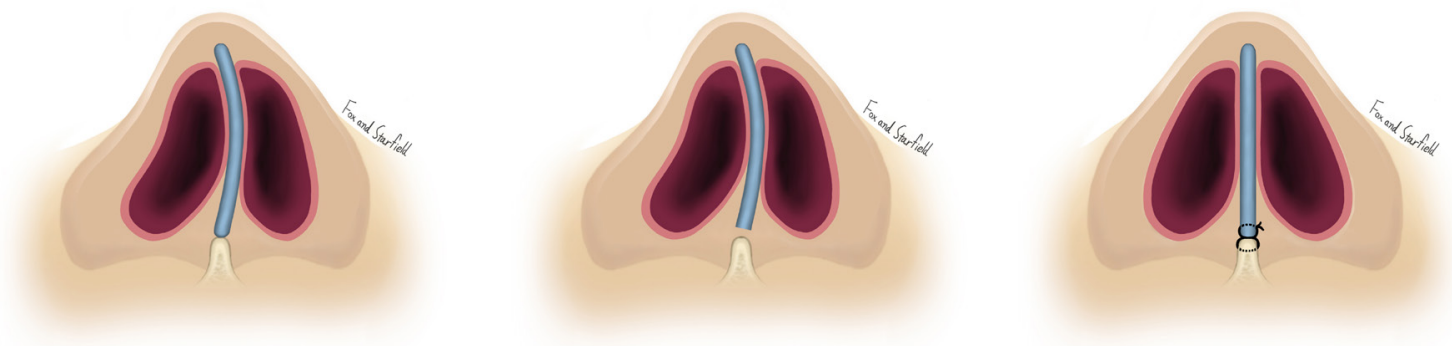

Fig. 14. Correction of a deviated caudal septum

(A) This patient had columellar deviation and nostril asymmetry. (B) The caudal septum was deviated to the left, and the posterior end of the septum was placed on the anterior nasal spine. (C) The excess caudal septum was shortened with segmental resection. (D) The caudal septum became straight after shortening. (E) The nasal tip and columella were at the midline, with symmetric nostrils after surgery.
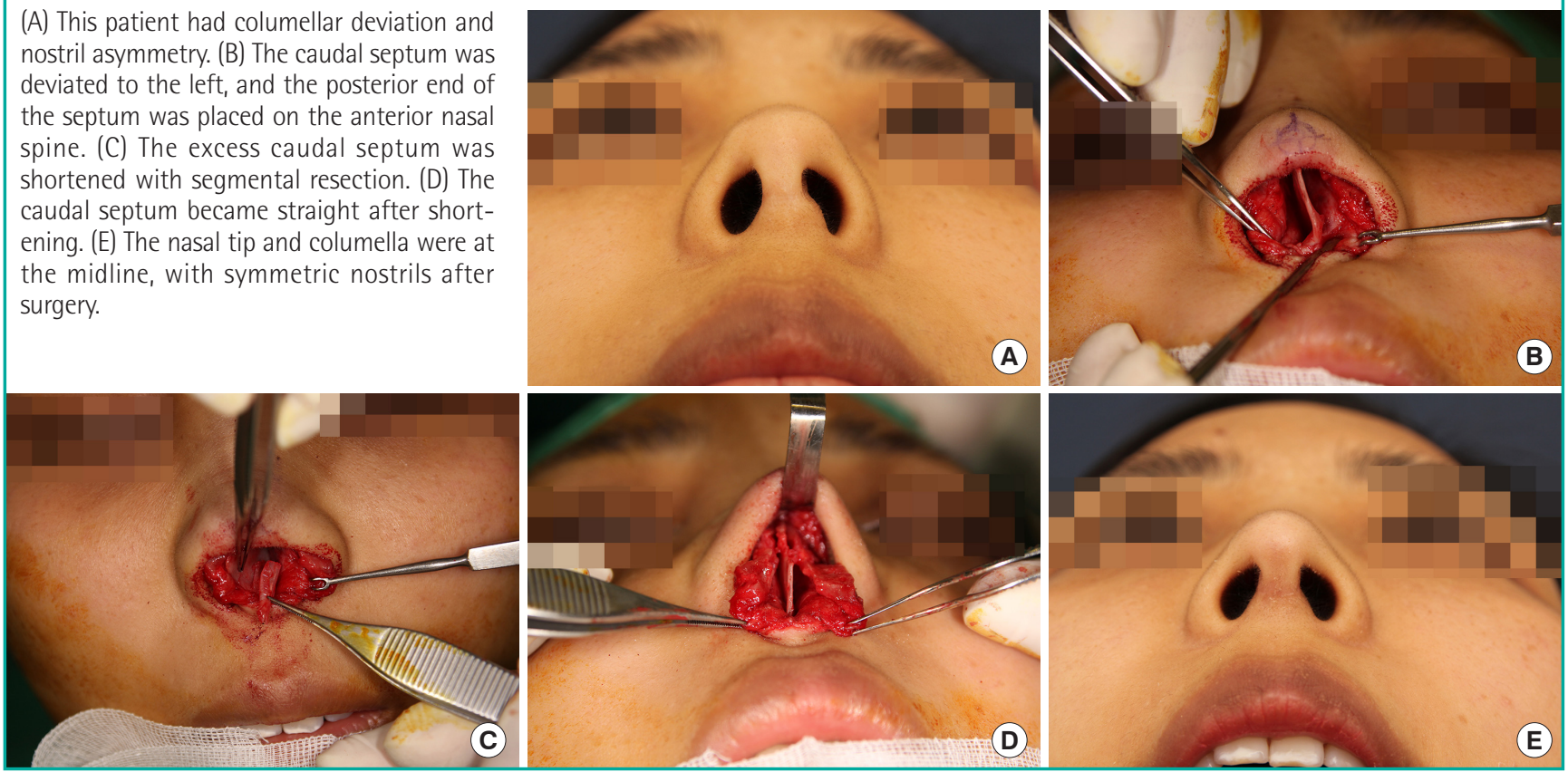

A splinting graft using removed septal cartilage for submucosal resection (SMR) should be applied to provide more stable support on the concave side [8].

The type with dislocation of the caudal septum from the maxillary crest and the ANS

At its posterior end, the caudal septum is segmentally resected and fixed to the periosteum of the ANS (Fig. 16) [2]. In order to do this, the ANS should be located in the midline.

If the ANS is located elsewhere, there are two ways to solve this problem. One way is to reposition the shortened caudal septum and fix it to the side of the ANS, not on the ANS (Fig. 17) [9]. Another method is repositioning it in the midline after osteotomy of the ANS in a greenstick fracture type [10].

After surgery using the abovementioned methods, the septum must be kept straight by applying an intranasal extramucosal silicone splint for 1-2 weeks.

\section{Correction of deviation of the dorsal septum}

After SMR is performed and deviation of the caudal septum is corrected, deviation of the dorsal septum improves spontaneously. The choice of an operative technique is made after examining the shape and extent of the remaining dorsal septal deviation.

The surgical methods to correct dorsal septal deviation are double mattress sutures, scoring and splinting grafts [11], the cut-and-suture technique, spreader grafts [12], and dorsal septal rotation sutures (clocking sutures) [13]. Most dorsal septal correction procedures are performed by combining multiple of the aforementioned methods.

The operative technique that the author prefers is a combination of a scoring and splinting graft (which additionally provides the functionality of a spreader graft) and clocking sutures.

\section{Scoring and splinting grafts}

The dorsal septal cartilage is moved to the midline by perform- 
Fig. 15. Cut-and-suture technique for a deviated caudal septum

$(A, B)$ This female patient had a deviated tip/ columella and nostril asymmetry. (C) On exploration, the right medial crus was longer than the left. (D) The caudal septum was deviated to the left. (E) Illustration of the cut-andsuture technique. (F) The caudal septum was straightened after the cut-and-suture technique. (G) The right medial crus was shortened and the columellar strut graft was placed. $(H, I)$ Postoperatively, the tip and columella were no longer deviated, and the nostrils were symmetric.
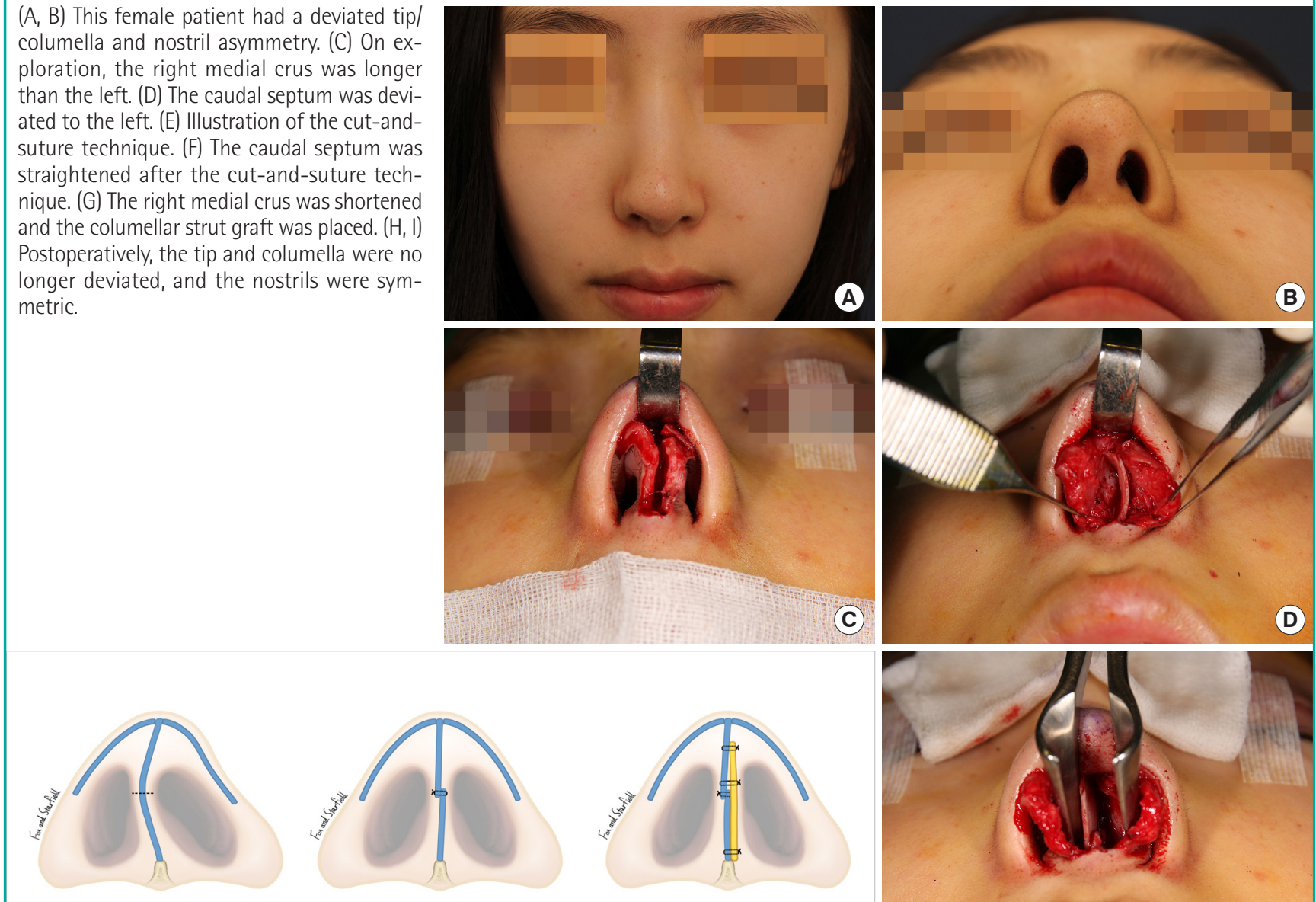

(E)
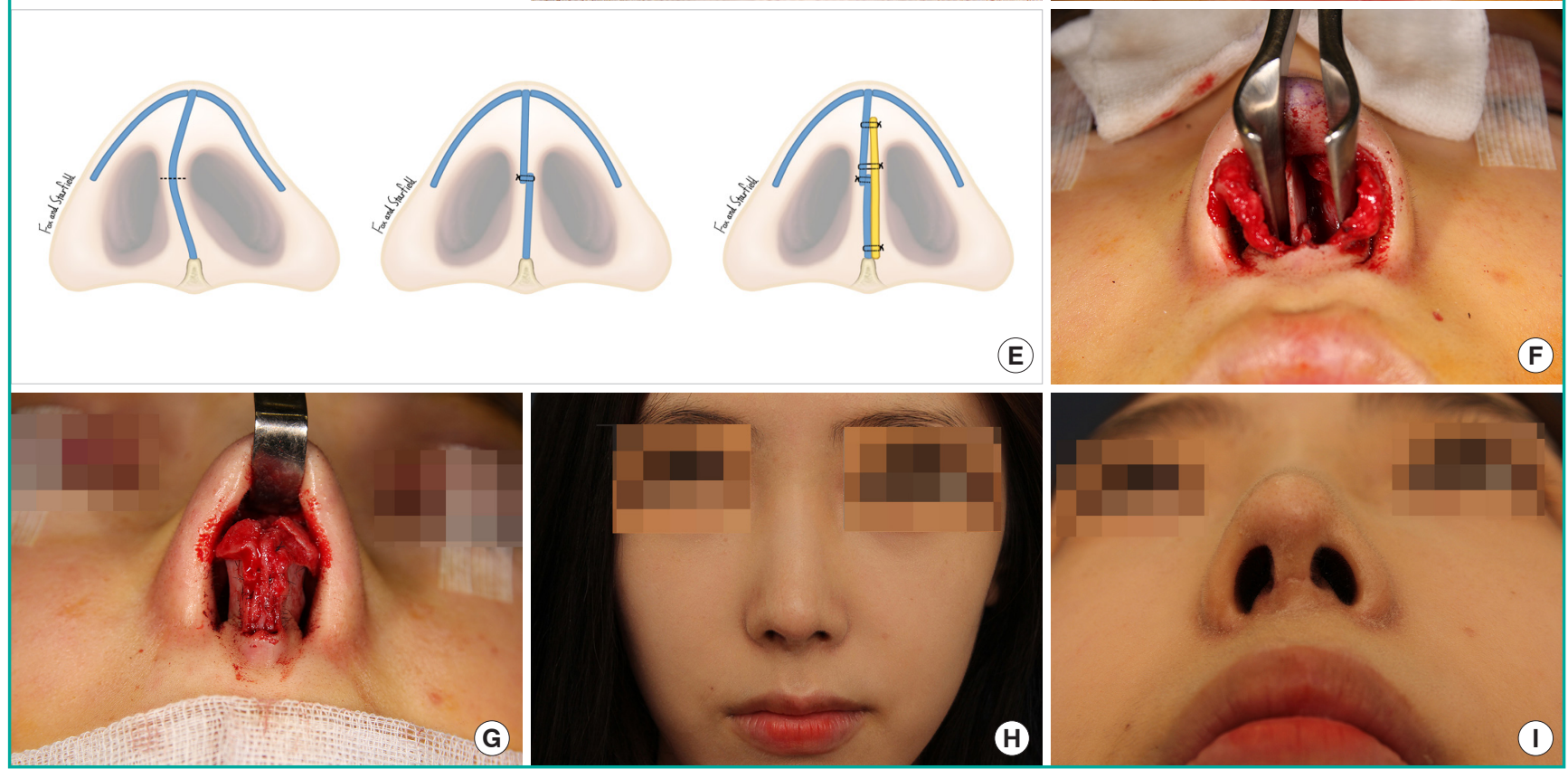

Fig. 16. Shortening of the caudal septum
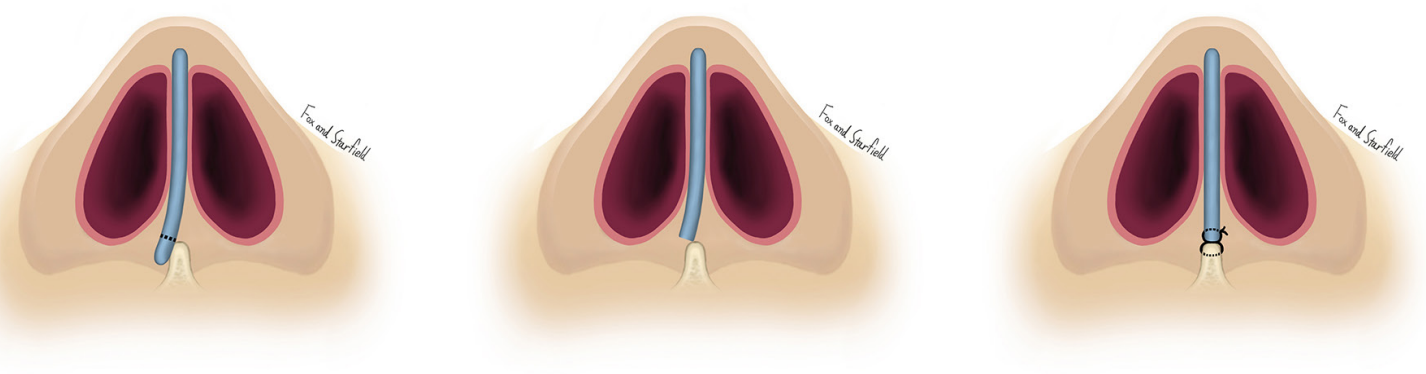


\section{Fig. 17. Displaced anterior nasal spine}

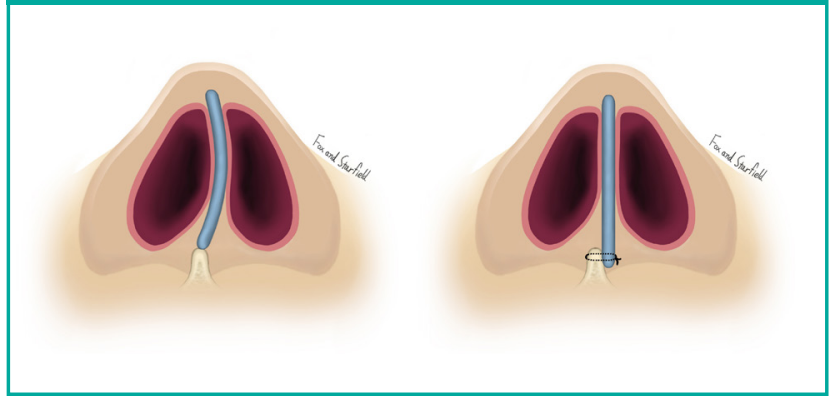

Fig. 18. Dorsal septal scoring and splinting graft

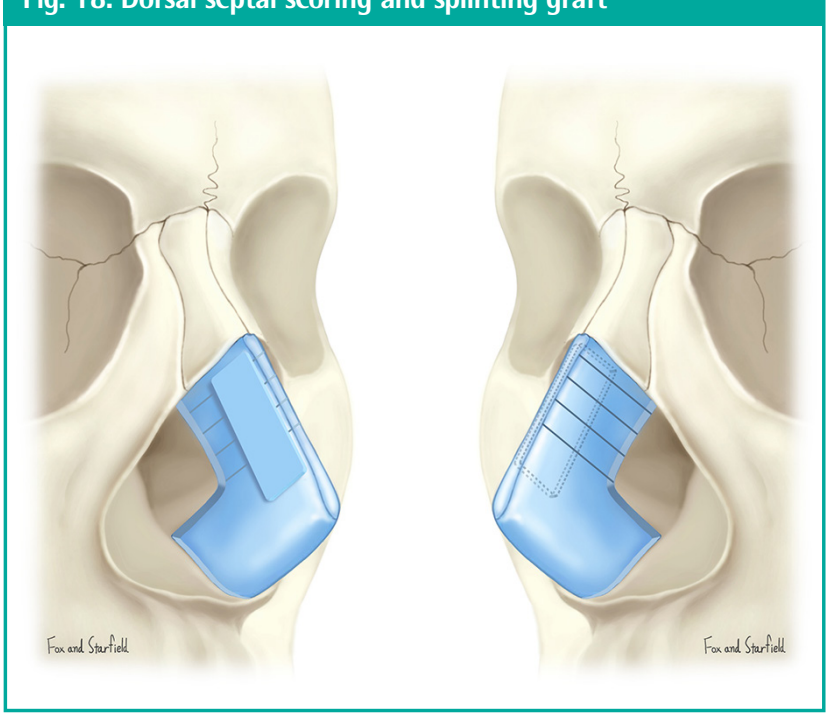

\section{Fig. 20. Clocking suture (dorsal septal rotation suture)}

ULC, upper lateral cartilage.
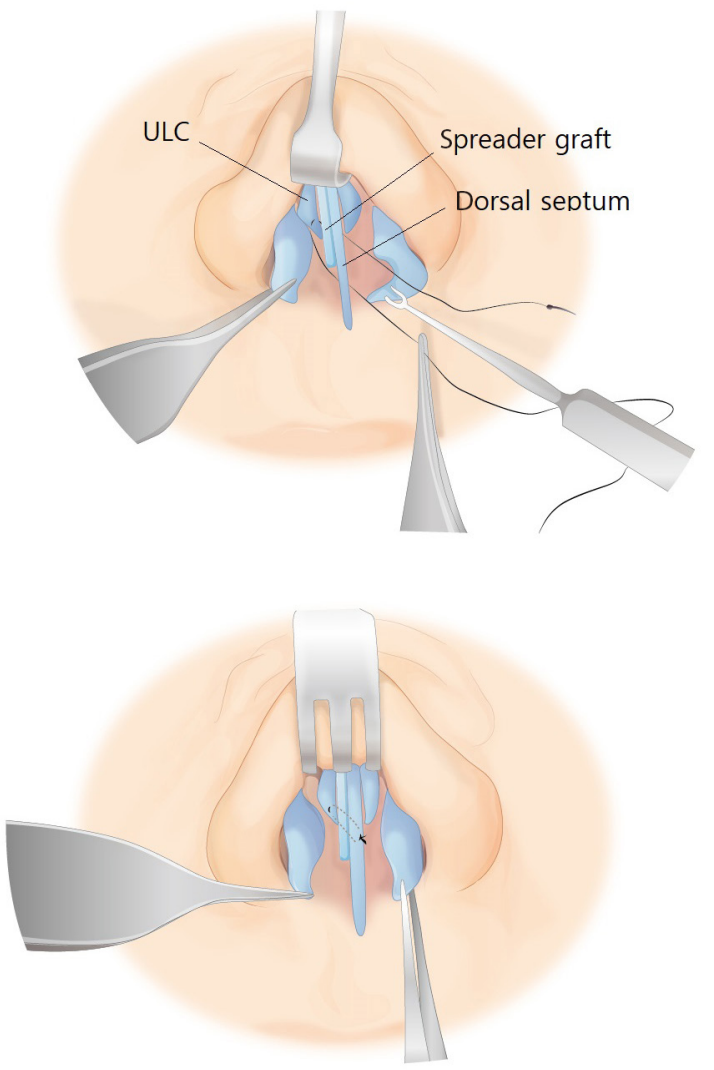

Fig. 19. Shaving the transverse wing of dorsal septum
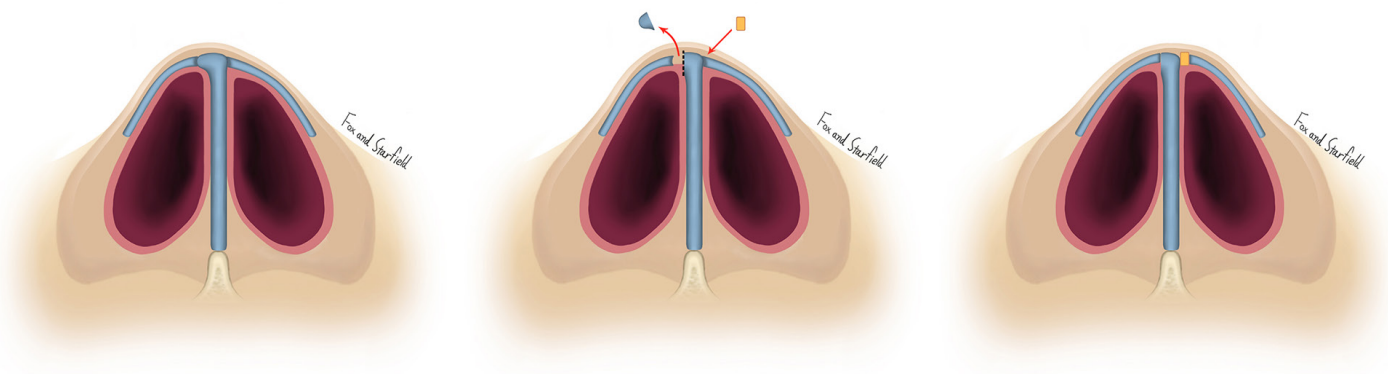

ing one-third-thick multiple scoring on the convex side of the dorsal septum [11]. Since the predictability is low and the dorsal septum is only weakened by scoring, a splinting graft is performed on the opposite side of the scored site (Fig. 18). The splinting graft additionally provides the functionality of a spreader graft. The spreader graft is a very important technique that is almost always included in dorsal septal correction, and it is usually located on the concave side. The spreader graft straightens the deviated dorsal septum and spreads the sunken supper lateral cartilage laterally. Moreover, it strengthens the dorsal septum weakened by scoring or the wedge resection, enables the prevention or correction of an inverted V-deformity, and increases the width of the internal nasal valve. The septal cartilage is the optimal donor site, but it is also possible to use the auricular cartilage or costal cartilage.

The sunken area is spread out by a spread graft on the concave side of the dorsal septum, whereas the transverse wing is resected if the convex side of the dorsal septum shows excessive concavity (Fig. 19) [11]. 


\section{Fig. 21. Correction of deviated dorsal septum}

$(A, B)$ This patient had a deviated dorsum and caudal septum. (C, D) At 5 months after nasal bone osteotomy, spreader graft, shaving of transverse wing of the left dorsal septum, and a clocking suture, correction of dorsal deviation, tip/columella deviation, and nostril asymmetry had been achieved.
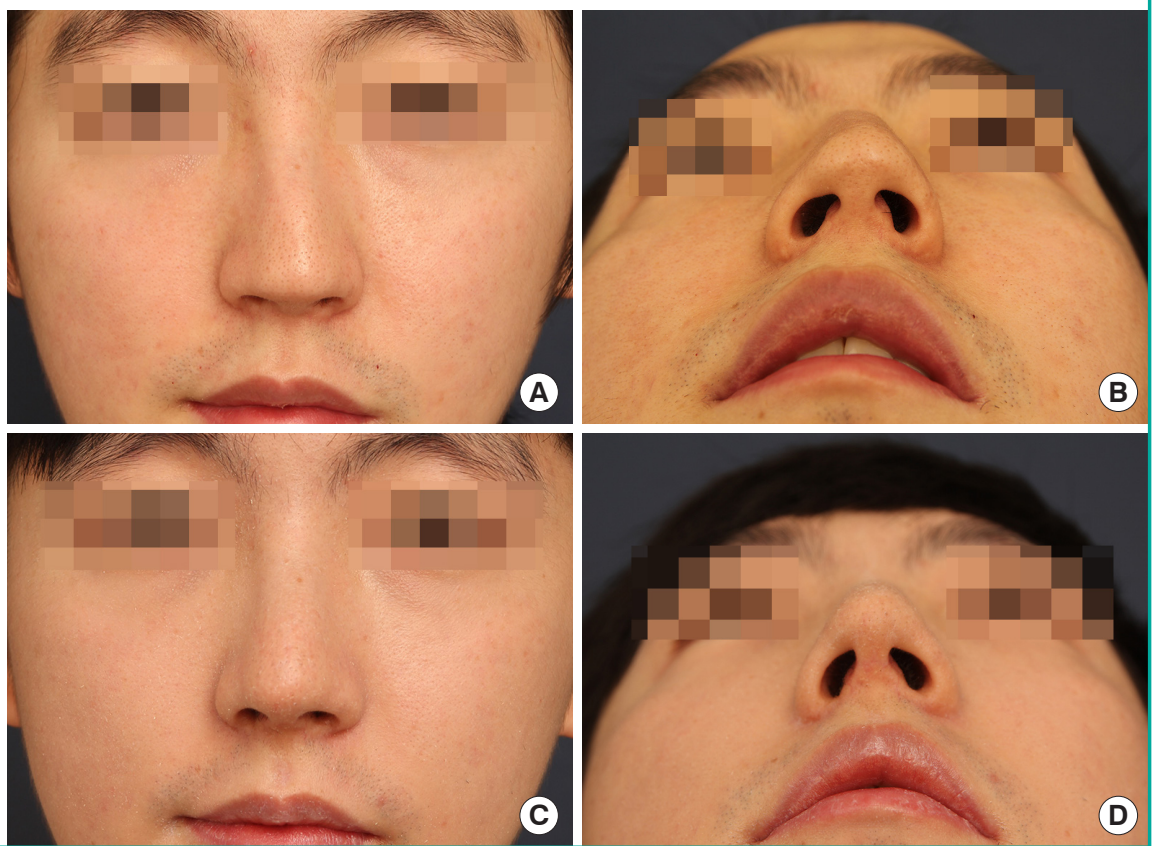

Fig. 22. Correction of an asymmetric alar rim

$(A, B)$ Patient with a deviated nose and retracted ala on the left side. $(C, D)$ The left retracted alar rim was corrected with an alar rim graft.
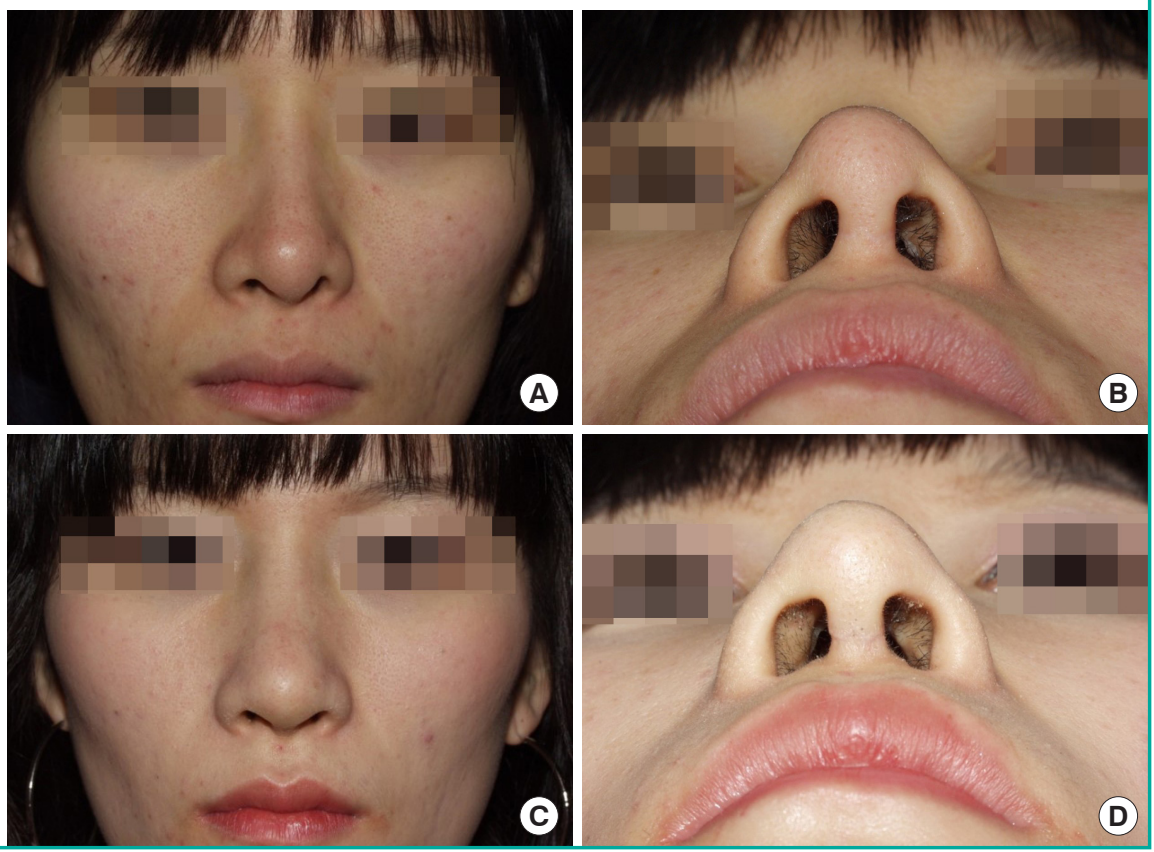

\section{Clocking suture (septal rotation suture)}

A clocking suture is a horizontal mattress suture that is anchored at the more cephalic portion of the upper lateral cartilage that passes through the more caudal part of the deviated dorsal septum and the spreader graft [13]. Thus, this suture can pull the deviated dorsal septum toward the midline (Fig. 20). The opposite upper lateral cartilage is then sutured to the straightened dorsal septum.
Fig. 21 presents a case where dorsal septal deviation, tip and columella deviation, and asymmetry of the nostrils were corrected by using a combination of the abovementioned three surgical methods.

\section{Correction of an asymmetric alar rim and alar base}

An asymmetric alar rim (retracted alar rim) or alar base asymmetry can cause nostril asymmetry in a deviated nose. Correc- 
tion of these factors should not be overlooked in order to achieve tip and nostril symmetry when correcting a deviated nose.

The correction methods of a retracted alar rim are caudal rotation of the lower lateral cartilage, alar rim grafts, lateral crural strut grafts, and composite grafts (Fig. 22) [14-16].

Normally, a width asymmetry of the alar base can be easily corrected by reduction of the wider side.

\section{CONCLUSION}

The correction of a deviated nose is not only limited to the correction of the nasal framework. If correction of nasal tip and columella deviation and asymmetry of the nostrils is not completed, it is difficult to receive a good evaluation from patients who undergo deviated nose correction.

Deviation of the tip and columella and asymmetry of the nostrils are not limited simply to lower lateral cartilage problems. The more important underlying etiology is caudal and dorsal septal deviation, which is hidden behind the deviated tip and columella. Therefore, to achieve good results, a surgeon should focus on correcting the deviated caudal and dorsal septum, as well as lower lateral cartilage deformation. Furthermore, correcting asymmetries of the alar rim and alar base is also important.

\section{NOTES}

\section{Conflict of interest}

No potential conflict of interest relevant to this article was reported.

\section{Patient consent}

The patients provided written informed consent for the publication and the use of their images.

\section{ORCID}

Man-Koon Suh

https://orcid.org/0000-0001-7586-1858

\section{REFERENCES}

1. Rohrich RJ, Gunter JP, Deuber MA, et al. The deviated nose: optimizing results using a simplified classification and algorithmic approach. Plast Reconstr Surg 2002;110:150923.

2. Guyuron B, Uzzo CD, Scull H. A practical classification of septonasal deviation and an effective guide to septal surgery. Plast Reconstr Surg 1999;104:2202-9.

3. Suh MK, Jeong E. Correction of deviated nose. Arch Craniofac Surg 2018;19:85-93.

4. Suh MK. Deviated nose correction and functional rhinoplasty. In: Suh MK, editor. Atlas of Asian rhinoplasty. Singapore: Springer Publishing Company; 2018. p. 735-9.

5. Momeni A, Gruber RP. Primary open rhinoplasty. Aesthet Surg J 2016;36:983-92.

6. Gruber RP, Chang E, Buchanan E. Suture techniques in rhinoplasty. Clin Plast Surg 2010;37:231-43.

7. Guyuron B, Behmand RA. Caudal nasal deviation. Plast Reconstr Surg 2003;111:2449-57.

8. Jang YJ, Yeo NK, Wang JH. Cutting and suture technique of the caudal septal cartilage for the management of caudal septal deviation. Arch Otolaryngol Head Neck Surg 2009; 135:1256-60.

9. Constantine FC, Ahmad J, Geissler P, et al. Simplifying the management of caudal septal deviation in rhinoplasty. Plast Reconstr Surg 2014;134:379e-388e.

10. Rengaraja D, Jagade M, Kale V, et al. As the nasal spine goes, so goes the septum. J Clin Diagn Res 2016;10:MC04-6.

11. Suh MK. Deviated nose correction and functional rhinoplasty. In: Suh MK, editor. Atlas of Asian rhinoplasty. Singapore: Springer Publishing Company; 2018. p. 707-24.

12. Ahmad J, Rohrich RJ. The crooked nose. Clin Plast Surg 2016;43:99-113.

13. Keeler JA, Moubayed SP, Most SP. Straightening the crooked middle vault with the clocking stitch: an anatomic study. JAMA Facial Plast Surg 2017;19:240-1.

14. Gruber RP, Fox P, Peled A, et al. Grafting the alar rim: application as anatomical graft. Plast Reconstr Surg 2014;134: 880e-887e.

15. Gunter JP, Friedman RM. Lateral crural strut graft: technique and clinical applications in rhinoplasty. Plast Reconstr Surg 1997;99:943-52.

16. Tardy ME Jr, Toriumi D. Alar retraction: composite graft correction. Facial Plast Surg 1989;6:101-7. 